\title{
Estrés, Depresión Y Consumo De Alcohol En Estudiantes De Bachillerato De México: Diferencias Por Sexo Y Escuela
}

\author{
Rosa Vargas Almendra, Licenciada \\ Arturo del Castillo Arreola, Doctor \\ Rebeca María Elena Guzmán Saldaña, Doctora
}

Universidad Autónoma del Estado de Hidalgo

doi: 10.19044/esj.2016.v12n32p94 URL:http://dx.doi.org/10.19044/esj.2016.v12n32p94

\begin{abstract}
Stress in teenagers studying high school is a health problem of great importance as not only affects behavior but also affects physical and mental health of this population, increasing the likelihood of depression occur and use alcohol as a means of coping with it. Objective: To determine the interaction by sex and type of school, as well as the relationship between levels of stress, depression and alcohol of high school students in two states in Mexico. Material and Methods: 129 students from two high schools in the states of Hidalgo and Mexico respectively selected through a non-probability sampling. The age range was from 16 to $21, \mathrm{M}=17$ years, $\mathrm{SD}=.893 .51 .2 \%$ were men and $48.8 \%$ women. Perceived Stress Scale (EEP) was used, the Beck Depression Inventory and Identification Questionnaire disorders due to alcohol consumption (AUDIT). They were conducted descriptive analyzes, Pearson correlation analysis and factorial ANOVA 2x2. Results: Significant negative relationships between stress and depression $(\mathrm{r}=.552, \mathrm{p}<0.05)$ were found, also significant differences by gender in alcohol $\mathrm{F}(3,70)=3.283, \mathrm{p} \leq$ .05 and significant differences by school in depression variable $\mathrm{F}(3,125)=$ $3.690, \leq .05$. Conclusion: These results provide insight into the association between different risk facotres student population so that from it culturally relevant psychological interventions that affect the prevention of health problems in this population are designed.
\end{abstract}

Keywords: Stress, depression, alcohol, students, high school

\section{Introducción}

El estrés constituye uno de los factores más estudiados en la actualidad, puesto que está asociado con diferentes enfermedades y en 
diferentes poblaciones, siendo los estudiantes uno de los grupos más afectados.

De acuerdo con The American Institute of Stress (AIS, 2012), tres de cada cuatro visitas al doctor son por dolencias relacionadas con el estrés, el 44\% de los estadounidenses que padecen estrés reporta que tienen problemas para dormir todas las noches y el $40 \%$ come en exceso o consume alimentos poco saludables, por lo que estar expuesto a eventos estresantes por periodos prolongados incrementa un $40 \%$ el riesgo de padecer enfermedades del corazón, 25\% el riesgo de sufrir un ataque al corazón y 50\% el riesgo de derrame cerebral; lo que convierte al estrés en la principal causa del 60\% de las enfermedades.

De acuerdo con una encuesta realizada por la Asociación Americana de Psicología (APA, 2014) los adolescentes durante periodos escolares mantienen un nivel de estrés más alto que el reportado por los adultos, de los cuales el $31 \%$ de los estudiantes manifestó sentirse abrumados, el 30\% deprimidos o tristes, el 36\% expresó sufrir fatiga o sentirse cansados y el $26 \%$ informó haberse saltado una comida como resultado del estrés; el estudio se llevó a cabo en Estados Unidos con la participación de 1,950 adultos mayores de 18 años y 1,018 adolescentes de entre 13 y 17 años.

Lazarus y Folkman (1984) mencionan que el estrés es el resultado de una relación entre el sujeto y el entorno en el que se desenvuelve el cual el individuo lo evalúa como amenazante y esto conlleva a poner en riesgo su bienestar. De acuerdo con estos autores, el estrés aparece cuando hay un desequilibrio entre la percepción que se tiene de las exigencias y la habilidad que tiene el individuo para cumplir dichas exigencias, de esta manera, la reacción que se tenga ante una situación estresante dependerá de la evaluación que realice la persona de la situación, la cual puede ser vista como irrelevante o amenazante, y de la evaluación de sus habilidades que posee para controlar o cambiar esa situación.

Los síntomas del estrés se dividen en físicos: problemas digestivos, dolores de cabeza, insomnio, cansancio, taquicardia, aumento de la presión arterial, aumento de la actividad respiratoria, hiper-sudoración, dilatación pupilar cuello o mandíbula rígidos; psicológicos: irritabilidad, ansiedad o ataques de pánico, tristeza o depresión, problemas de concentración, disminución de rendimiento intelectual, desorientación temporo-espacial; y conductuales: Imposibilidad para relajarse, beber en exceso, comer en exceso, fumar o usar drogas (National Institute of Mental Health [NIMH, 2012]; Maturana \& Vargas, 2015).

El estrés puede ser positivo (eustrés) el cual se refiere a la cantidad de tensión necesaria para llevar una vida activa y saludable y que nos permite escapar del peligro, o puede ser negativo (distrés) el cual surge a causa de tensiones que pueden conducir a la enfermedad física; va a depender de la 
persona y la manera en que tome las demandas del día a día y por ende de sus propias capacidades para hacerles frente (Alawad \& Slamah, 2014).

Estar expuesto a eventos estresantes por periodos prolongados de tiempo puede causar ansiedad, insomnio, dolor muscular, presión alta, enfermedades del corazón, diabetes, depresión, ansiedad y debilitamiento del sistema inmunitario (APA, 2014).

A pesar de su relevancia son pocos los estudios en Latinoamérica que han abordado el estrés en estudiantes, siendo aún más escasos en estudiantes de nivel medio superior. Entre aquellos que se han publicado, existen algunos que se han centrado en como el estrés afecta a esta población; algunos de ellos refieren que ante situaciones estresantes y la falta de estrategias de afrontamiento para el manejo de problemas los estudiantes deciden consumir alcohol para afrontar, olvidar o escapar de situaciones difíciles de la vida, problemas o crisis personales (Flórez \& Trujillo, 2013).

En un estudio llevado a cabo en estudiantes universitarios encontró que los factores académicos son la principal causa de estrés en esta población y el estresor más frecuente fue la sobrecarga de trabajo, por encima de factores personales y factores ambientales (Alawad \& Slamah, 2014).

Pozos-Radillo, Preciado-Serrano, Plascencia, Acosta-Fernández y Aguilera (2015), mencionan que algunos eventos que están relacionados con el estrés en estudiantes son presentaciones de resultados o exámenes, participación frente a compañeros o trabajos en grupo, así como tener tiempo limitado para tareas o no entender los temas de la clase, por lo que el tener un buen rendimiento académico está relacionado con las estrategias de afrontamiento que poseen los estudiantes para mantener niveles bajos de estrés.

Por otro lado, los programas de estudio del nivel medio superior demandan de los alumnos gran responsabilidad y dedicación, generando altos niveles de estrés, afectando de manera negativa el proceso de aprendizaje y memoria (García \& Escalera, 2011).

Debido a su alta prevalencia y al impacto que tiene sobre los estudiantes de bachillerato, el estrés académico se ha convertido en un problema de salud pública (Reyes, Ibarra, Torres \& Razo, 2012) afectando su rendimiento académico y generando importantes consecuencias en la salud de esta población como son: la presencia de estados depresivos, ansiedad, irritabilidad, descenso de la autoestima, insomnio, asma, hipertensión, úlceras y consumo de sustancias nocivas (Berrío \& Mazo, 2011; Khurshid, Parveen, Yousuf \& Ghafoor, 2015).

Dentro de las variables que se asocian con el estrés se encuentra la depresión, cuyos síntomas de acuerdo con el Manual Diagnóstico y Estadístico de los Trastornos Mentales, quinta edición ([APA], 2014) incluyen: 
1) Estado de ánimo deprimido la mayor parte del día, casi todos los días

2) Disminución del interés o placer por todas o casi todas las actividades la mayor parte del día, casi todos los días

3) Pérdida importante de peso sin hacer dieta o aumento de peso, sí como disminución o aumento del apetito

4) Insomnio o hipersomnia

5) Agitación o retraso psicomotor

6) Fatiga o pérdida de energía

7) Sentimiento de inutilidad o culpabilidad excesiva o inapropiada

8) Disminución de la capacidad para pensar o concentrarse, o tomar decisiones

9) Pensamientos de muerte recurrentes, ideas suicidas recurrentes sin un plan determinado, intento de suicidio o un plan específico para llevarlo a cabo

De acuerdo con la intensidad de los síntomas los episodios pueden ser (Alcalá, 2014):

1) Leves, cuando el paciente tiene pocos síntomas y estos no alteran su actividad diaria

2) Moderados, cuando hay un mayor número de síntomas o de mayor intensidad limitando sus funciones y generando un gran esfuerzo por cumplir con sus labores y

3) Severos donde el paciente tiene todos los síntomas depresivos y se siente ilimitado o imposibilitado de realizar sus actividades diarias

La depresión es la principal causa de discapacidad mundial, contribuyendo así a una considerable carga mundial de morbilidad y en el peor de lo casos puede llevar al suicidio, que es la causa de aproximadamente un millón de muertes anuales. Se calcula que alrededor de unos 350 millones de personas en todo el mundo se han visto afectadas por esta enfermedad, sin embargo, no siempre se recibe tratamiento debido a la escasez de recursos y a la falta de personal capacitado (Organización Mundial de la Salud [OMS], 2012).

Existe mayor prevalencia de depresión en mujeres que en hombres, ya que en la actualidad los jóvenes de entre 15 y 24 años están más expuestos a situaciones que ponen en riesgo su salud física y mental (Cubillas, Román, Valdez \& Galaviz, 2012; Centro de Estudios para el Adelanto de las Mujeres y la Equidad de Género CEAMEG] ,2013).

Los efectos de la depresión en estudiantes van desde presentar síntomas somáticos como son alteraciones del sueño, dolores de espalda, cansancio, así como problemas conductuales y cognitivos como irritabilidad, falta de concentración baja motivación y sensación de fracaso hasta agresividad (Soria, Ávila \& Morales, 2014). 
Con respecto a la asociación entre estrés y depresión, se ha encontrado que los sucesos vitales estresantes que experimentan los estudiantes están relacionados significativamente con síntomas de depresión, por lo que a mayor nivel de estrés mayor nivel de depresión en los estudiantes (Veytia, González \& Andrade, 2012; González-Olaya, DelgadoRico, Escobar-Sánchez \& Cárdenas-Angelone, 2014; Iqbal, Gupta \& Venkatarao, 2015).

Otra de las variables asociadas con el estrés, es el consumo de alcohol, que afecta a las personas y a las sociedades de diferentes maneras, y sus efectos están determinados por la cantidad de alcohol consumido; además de que está asociado con el riesgo de desarrollar problemas de salud tales como el alcoholismo, la cirrosis hepática, algunos tipos de cáncer, enfermedades cardiovasculares, así como traumatismos derivados de la violencia y los accidentes de tránsito (OMS, 2015).

Para que a una persona se le considere con el Síndrome de Dependencia al Alcohol (Aguillón, 2014) se deben de cumplir al menos tres de los siguientes siete síntomas:

1) tolerancia notable a las bebidas alcohólicas

2) síntomas de abstinencia y consumo de alcohol para aliviar la abstinencia

3) consumo en mayor cantidad de alcohol o durante más tiempo

4) Deseos persistentes o esfuerzos inútiles para dejar de consumir o para controlar el consumo de alcohol

5) uso de gran parte de su tiempo para conseguir la sustancia

6) abandono de sus actividades y planes cotidianos

7) consumo continuo de alcohol a pesar de tener problemas físicos y psicológicos relacionados con la bebida

En 2011 la Encuesta Nacional de Adicciones [ENA], reportó que 4.9 millones de personas en México de entre 12 y 65 años es decir el seis por ciento de la población desarrollo dependencia al alcohol, siendo el 1.8 por ciento mujeres y el 10.8 hombres (Instituto Nacional de Psiquiatría Ramón de la Fuente Muñíz [INPRFM], 2012).

De acuerdo con la Encuesta Nacional de Salud y Nutrición (ENSANUT, 2012), la prevalencia de consumo de alcohol en adolescentes es de $28.8 \%$ en los hombres y $21.2 \%$ en las mujeres, mientras que en los adultos mayores de 20 años es de $67.8 \%$ en hombres y $41.3 \%$ en mujeres.

Por otro lado, el consumo de alcohol en jóvenes estudiantes es una preocupación social debido a que aumenta la probabilidad de presentar un consumo problemático además de que está asociado con conductas delictivas, siendo los hombres los principales consumidores (Uribe, Verdugo \& Zacarías, 2011; Armendáriz, Villar, Alonso, Alonso \& Oliva, 2012). 
El consumo de alcohol representa uno de los principales problemas de salud, sobre todo en estudiantes debido a que está asociado a diversos factores, que hacen referencia a emociones desagradables, momentos agradables con otros, búsqueda de situaciones nuevas y situaciones de conflicto con padres, en las que los jóvenes recurren al consumo de sustancias nocivas para reducir el estrés y la tensión que provocan estas situaciones y de esta manera conseguir un estado de bienestar (Palacios, 2012; Mphele, Gralewski \& Balogun, 2013).

La relación entre los eventos estresantes y el consumo de alcohol es muy común en estudios sobre estudiantes debido a que estos lo utilizan como un medio de afrontamiento dado que enfrentan diversas situaciones físicas, emocionales y sociales con el fin de mitigar tales situaciones (TelumbreTerrero, Esparza-Almanza, Alonso-Castillo \& Alonso-Castillo, 2016).

En un estudio realizado en estudiantes, se encontró que los eventos estresantes y el consumo de alcohol se relacionan positiva y significativamente, por lo que a mayor nivel de estrés, mayor consumo de alcohol en estudiantes (Armendáriz et al., 2012; Tovolacci et al., 2013).

El estrés es un fenómeno que ha impactado a lo largo del tiempo aumentando el número de casos por año, además de que trae consigo daños a nivel psicológico, físico y social, sobre todo a los estudiantes, es por ello la importancia de estudiar la relación entre el nivel de estrés y los factores psicosociales que repercuten en el desarrollo de dichos jóvenes con el fin de generar un programa de intervención psicológica que beneficie a esta población.

El objetivo de este estudio fue determinar la interacción por sexo y por tipo de escuela, así como la relación entre los niveles de estrés, depresión y consumo de alcohol de jóvenes adultos del Estado de Hidalgo y Estado de México.

Como hipótesis se plantea que existe interacción por sexo y tipo de escuela, así como relación estadísticamente significativa entre los niveles de estrés, depresión y consumo de alcohol de jóvenes adultos del Estado de Hidalgo y Estado de México.

\section{Método}

\section{Participantes}

Participaron 129 estudiantes del sexto semestre de nivel medio superior del CECyTEH plantel Epazoyucan y la preparatoria 37 del estado de Hidalgo y estado de México respectivamente seleccionados mediante un muestreo no probabilístico. El rango de edad osciló de los 16 a los 21 años, la media de edad fue de 17 años con una desviación estándar de .893. El $51.2 \%$ fueron hombres y el $48.8 \%$ mujeres. 


\section{Instrumentos}

Para medir el estrés se utilizó la Escala de Estrés Percibido, instrumento construido y validado por González y Landero (2007). La versión utilizada consta de 14 ítems con puntuación de nunca (0) a muy a menudo (4). La mayor puntuación corresponde a mayor estrés percibido. El instrumento cuenta con una consistencia interna alfa de Cronbach de .83.

Para medir depresión se utilizó el Inventario de Depresión de Beck versión corregida y estandarizada por Jurado et al. (1998) en población mexicana. Consta de 21 ítems tipo Likert con cuatro opciones de respuesta (0-3). Se presentan ordenadas de menor a mayor gravedad y cuenta con una consistencia interna alfa de Cronbach de.87

Para medir consumo de alcohol se utilizó el Cuestionario de Identificación de los Trastornos debidos al consumo de Alcohol (AUDIT) desarrollado por la Organización Mundial de la Salud, consta de 10 reactivos que evalúan la frecuencia y cantidad del consumo de alcohol, la dependencia al consumo de alcohol y el consumo de alcohol perjudicial o dañino. Cuenta con una consistencia interna de .85 .

\section{Procedimiento}

Se contactó con el director de ambas instituciones solicitando su apoyo con los permisos pertinentes para la aplicación de los instrumentos de medición, posteriormente se realizaron dos sesiones de aplicación por escuela de manera colectiva donde se les explico a los alumnos el objetivo de la investigación y la manera correcta de llenar los cuestionarios; después se llevó acabo la captura de los datos en el programa estadístico SPSS, con dicho programa se elaboró una base de datos. Por último, se llevaron a cabo análisis descriptivos, análisis de correlación de Pearson y análisis factorial de Varianza ANOVA 2x2.

\section{Resultados}

El objetivo del estudio fue determinar la interacción por sexo y por tipo de escuela, así como la relación entre los niveles de estrés, depresión y consumo de alcohol de jóvenes adultos del Estado de Hidalgo y Estado de México.

Respecto a la presencia de eventos estresantes se observa que los estudiantes tienen una ligera inclinación hacia el estrés positivo $(\mathrm{M}=17.71$, $\mathrm{DE}=4.15)$, en comparación al estrés negativo $(\mathrm{M}=14.16, \mathrm{DE}=4.98)$.

En cuanto a la distribución por nivel de depresión $(\mathrm{M}=10.30$, $\mathrm{DE}=8.16$ ), el $5.4 \%$ de los estudiantes tiene riesgo de padecer depresión severa, el $10.1 \%$ de los estudiantes presentan niveles moderados de depresión, el $28.7 \%$ de los estudiantes se encuentra dentro del nivel leve de 
depresión, mientras que el $55.8 \%$ de los estudiantes presentan niveles mínimos de depresión, esto quiere decir que no se encuentran en riesgo.

Respecto a las dimensiones de consumo de alcohol $(\mathrm{M}=7.8, \mathrm{DE}=$ 6.27), el 3.9\% de los estudiantes se encuentra en un nivel de dependencia al alcohol lo cual indica que deben ser derivados con un especialista, el $4.7 \%$ de los estudiantes se encuentra en un nivel perjudicial de consumo, el 14\% de los estudiantes presenta un bajo riesgo al consumo perjudicial, mientras que el $77.5 \%$ de los estudiantes son abstemios, esto quiere decir que no se encuentran en riesgo.

Se utilizó el Coeficiente de Correlación de Pearson para determinar la relación entre el nivel de estrés, el nivel de depresión y el nivel de consumo de alcohol, dichos resultados se presentan en la tabla 1.

Tabla 1. Correlación de las dimensiones estrés, depresión y consumo de alcohol.

\begin{tabular}{ccc}
\hline & Depresión & Consumo de alcohol \\
\hline Estrés positivo & $-.388^{* *}$ & -.117 \\
\hline Estrés negativo & $.552^{* *}$ & .095 \\
\hline Depresión & & .004 \\
\hline & ${ }^{*} \mathrm{p}<.05 \quad{ }^{* *} \mathrm{p}<.01$ &
\end{tabular}

Se encontró una relación negativa estadísticamente significativa ( $\mathrm{r}=$ .388; $\mathrm{p}<0.05$ ) entre estrés positivo y depresión. Lo que indica que a mayor estrés positivo, menor será la probabilidad de padecer depresión. De igual manera se encontró relación positiva estadísticamente significativa $(r=.552$; $\mathrm{p}<0.05$ ) entre estrés negativo y depresión. Lo que indica que a mayor índice de estrés negativo, mayor será el nivel de depresión. Con lo que se comprueba que existe relación estadísticamente significativa entre el nivel de estrés y el nivel de depresión de jóvenes adultos del estado de Hidalgo y Estado de México.

Se encontró relación negativa entre estrés positivo y el consumo de alcohol $(\mathrm{r}=-.117 ; \mathrm{p}<0.05)$ lo que indica que a mayor estrés positivo, menor será la probabilidad de consumir alcohol, sin embargo esta relación no es significativa. De igual manera se encontró relación positiva $(r=.095 ; \mathrm{p}<0.05)$ entre estrés negativo y depresión. Lo que indicaría que a mayor índice de estrés negativo, mayor será el nivel de consumo de alcohol, sin embargo no es una relación estadísticamente significativa.

Se encontró relación positiva $(r=.004 ; \mathrm{p}<0.05)$ entre depresión y consumo de alcohol. Lo que indicaría que a mayor índice de depresión, mayor será el nivel de consumo de alcohol, sin embargo no es una relación estadísticamente significativa.

Para determinar la interacción por sexo y por tipo de escuela se llevó a cabo un análisis factorial de varianza ANOVA 2x2 cuyos resultados se encuentran en resumen en la Tabla 2. 
Tabla 2. Diferencia por sexo, escuela e interacción por sexo y carrera de las dimensiones de estrés, depresión y consumo de alcohol

\begin{tabular}{cccccccccc}
\hline & \multicolumn{3}{c}{ Sexo } & \multicolumn{3}{c}{ Escuela } & \multicolumn{3}{c}{ Sexo por escuela } \\
\hline & $\mathrm{F}$ & $\mathrm{gl}$ & Sig & $\mathrm{F}$ & $\mathrm{gl}$ & Sig & $\mathrm{F}$ & $\mathrm{Gl}$ & Sig \\
\hline Estrés positivo & 1.386 & 1 & .241 & .364 & 1 & .547 & 1.29 & 1 & .720 \\
Estrés negativo & 2.997 & 1 & .086 & .385 & 1 & .536 & .076 & 1 & .783 \\
Depresión & .146 & 1 & .703 & 4.952 & 1 & .028 & .118 & 1 & .732 \\
Consumo de alcohol & 4.634 & 1 & .035 & .263 & 1 & .609 & .574 & 1 & .451 \\
\hline
\end{tabular}

Por lo que respecta a la variable de estrés medidas con la escala de Estrés Percibido no se encontraron diferencias estadísticamente significativas por sexo, por escuela ni en la interacción de estas variables.

Existen diferencias estadísticamente significativas por escuela en las dimensiones de depresión $\mathrm{F}(3,125)=3.690, \leq .05$, mostrando que los alumnos del CECyTEH presentan promedios más altos que los estudiantes de la Preparatoria 37. No se encontraron diferencias por sexo ni en la interacción sexo por escuela.

Se encontraron diferencias estadísticamente significativas por sexo en las dimensiones de consumo de alcohol $\mathrm{F}(3,70)=3.283, \mathrm{p} \leq .05$, siendo los hombres los que presentan un mayor promedio en estas escalas. No se encontraron diferencias por tipo de escuela ni en la interacción entre sexo y tipo de escuela.

Tabla 3. Medias y desviación estándar por sexo y escuela de las dimensiones de los instrumentos de estrés, depresión y consumo de alcohol

\begin{tabular}{ccccc}
\hline & $\begin{array}{c}\text { Estrés } \\
\text { positivo }\end{array}$ & $\begin{array}{c}\text { Estrés } \\
\text { negativo }\end{array}$ & Depresión & $\begin{array}{c}\text { Consumo de } \\
\text { alcohol }\end{array}$ \\
\hline Sexo & & & & \\
\hline Mujeres & $18.26 \pm .592$ & $14.88 \pm .702$ & $10.18 \pm 1.14$ & $5.48 \pm 1.32$ \\
Hombres & $17.34 \pm .514$ & $13.27 \pm .609$ & $9.60 \pm .994$ & $8.97 \pm .936$ \\
\hline Escuela & & & & \\
\hline CECyTEH & $17.84 \pm 3.72$ & $13.57 \pm 3.78$ & $8.20 \pm 6.00$ & $9.06 \pm 7.00$ \\
Preparatoria 37 & $17.63 \pm 4.42$ & $14.54 \pm 5.62$ & $11.68 \pm 9.09$ & $6.67 \pm 5.39$ \\
\hline Mujeres CECyTEH & $18.65 \pm 3.62$ & $14.47 \pm 3.37$ & $8.24 \pm 4.49$ & $5.29 \pm 4.11$ \\
Mujeres Preparatoria & $17.89 \pm 4.05$ & $15.30 \pm 5.01$ & $12.13 \pm 8.10$ & $5.68 \pm 4.63$ \\
37 & & & & \\
Hombres CECyTEH & $17.44 \pm 3.75$ & $13.12 \pm 3.93$ & $8.18 \pm 6.69$ & $10.00 \pm 7.30$ \\
Hombres & $17.25 \pm 4.95$ & $13.44 \pm 6.31$ & $11.03 \pm 10.45$ & $7.94 \pm 6.14$ \\
Preparatoria 37 & & & &
\end{tabular}

\section{Conclusión}

El propósito de la investigación fue determinar la interacción por sexo y por tipo de escuela, así como la relación entre los niveles de estrés, 
depresión y consumo de alcohol de jóvenes adultos del Estado de Hidalgo y Estado de México.

En comparación con otros estudios esta muestra mantiene niveles de estrés menores que los encontrados en otros estudios al igual que en los niveles de depresión, sin embargo en comparación con otras muestras los estudiantes analizados en este estudio mantienen niveles más altos de consumo de alcohol.

En cuanto a la relación entre las variables estrés y depresión se encontró una relación positiva, lo que indica que a mayor nivel de estrés positivo, menor nivel de depresión; y a mayor nivel de estrés negativo, mayor nivel de depresión, estos resultados coinciden con los encontrados en un estudio llevado a cabo en estudiantes (Vega, Villagrán, Nava \& Soria, 2013; González-Olaya, Delgado-Rico, Escobar-Sánchez \& CárdenasAngelone, 2014). Puesto que los sucesos estresantes influyen en el estado físico y psicológico del individuo, y cualquier cambio o circunstancia diferente que se presente en la vida diaria y sea percibido como amenaza por la persona y además ésta sienta que no se está preparada física y psicológicamente para afrontarlo podría dar lugar a padecer algún tipo de depresión, debido a la acumulación de sentimientos ambiguos y respuestas contradictorias y frustrantes sobre las capacidades que se poseen para resolver la situación (Reyes, Ibarra, Torres \& Razo, 2012).

Por otro lado a mayor nivel de estrés positivo, menor nivel de depresión lo cual implica poner en marcha una serie de estrategias tanto conductuales como cognitivas enfocadas a la solución del problema a partir de la evaluación que se hace del evento estresante y que está relacionado con la capacidad para identificar los sentimientos y poder expresarlos, lo que a su vez facilita una mejor asimilación y adaptación ante situaciones de estrés, por lo que si la persona cree tener la capacidad de afrontar la situación sus niveles de depresión disminuirán (Chávez, Contreras \& Velázquez, 2014).

También se encontró una relación positiva entre el nivel de estrés y el consumo de alcohol, por lo que a mayor nivel de estrés mayor nivel de consumo de alcohol en estudiantes, esto coincide con los resultados de un estudio llevado a cabo en estudiantes (Armendáriz et al., 2012; Tovolacci et al., 2013). Esto puede deberse a que el consumo de alcohol reduce la respuesta fisiológica al estrés, cuando la persona carece de habilidades para controlar la situación estresante o para manejar sus propias emociones y buscar apoyo social, recurre al uso de esta sustancia la cual proporciona una experiencia placentera y positiva que le permite a la persona sentir que su ánimo mejora y al mismo tiempo le permite alejarse de sus preocupaciones, de esta manera, el alcohol se convierte en un distractor que le permite a la persona aliviar la sensación de malestar (Barnett, 2013; Michelini, Acuña \& Godoy, 2016). 
No se encontraron diferencias significativas por sexo en las dimensiones de estrés. Contario a lo que se ha encontrado en investigaciones anteriores donde las mujeres tienden a tener mayores niveles de estrés (García \& Escalera 2011; Veytia, González \& Andrade, 2012; Pozos-Radillo et al., 2015). Es posible que estos resultados se deban a una concepción diferente como lo es la igualdad de género, puesto que en años anteriores no se les permitía a los hombres expresar sus emociones ni realizar las mismas actividades que las mujeres debido a que era visto como signo de debilidad, sin embargo, hoy en día la sociedad les permite a los hombres expresarse abiertamente y demostrar cómo se sienten, por lo que cada vez más hombres optan por realizar las mismas actividades que las mujeres lo cual les genera mayores niveles de estrés y depresión. Por lo que tal vez las diferencias de género pueden jugar un papel más importante e influyan en los puntajes obtenidos donde no se encuentran diferencias significativas.

No se encontraron diferencias por sexo en las dimensiones de depresión, sin embargo, aunque son muchas las investigaciones que constatan la existencia de diferencias respecto a la presencia depresión entre hombres y mujeres, siendo las mujeres las que presentan niveles más altos, es importante señalar que las condiciones que hacen que tales diferencias se atenúen o aumenten aún están sujetas a debate debido a diferentes factores como la edad y la cultura (Matud, Correa, Bethencourt \& Del Pino, 2014); estos resultados coinciden con los encontrados en un estudio que analizó las diferencias de género sobre depresión en estudiantes, pues aunque las mujeres pueden ser más vulnerables a la depresión debido a factores psicosociales que les permiten expresar más libremente sus sentimientos, los hombres pueden estar expuestos a demandas sociales más exigentes como éxito escolar y mayores presiones económicas (Soria, Ávila \& Morales, 2014).

En cuanto a las diferencias por escuela se encontró que los alumnos de la Preparatoria 37 del Estado de México presentaron los puntajes mayores en las dimensiones de depresión, mientras que los alumnos del CECyTEH plantel Epazoyucan fueron los que presentaron menores puntajes, es posible que estos resultados se deban a que los estudiantes del CECyTEH están menos expuestos a factores estresantes debido a que la comunidad donde se encuentra la escuela es más pequeña con mayor interacción social y contacto con la naturaleza en comparación con los estudiantes de la Preparatoria 37 que se encuentran en una zona urbana, estos resultados coinciden con los encontrados en un estudio de depresión en estudiantes de México y Michoacán (Jiménez, Wagner, Rivera \& González-Forteza, 2015). Por lo que sería importante indagar más sobre las características sociodemográficas y su influencia en la salud mental. 
Con respecto a las diferencias por sexo, se encontró que los hombres consumen más alcohol que las mujeres. Estos resultados coinciden con un estudio llevado a cabo en estudiantes de bachillerato que muestra que las mujeres consumen alcohol con menos frecuencia que los hombres (González-González et al., 2012; Sandoval, Torres \& Martínez, 2015). En cuanto a las diferencias por escuela e interacción por sexo y escuela no se encontraron diferencias significativas ni otros estudios que corroboren estos resultados.

Es posible que estos resultados se deban a que el consumo de alcohol se ha asociado con factores de tipo social y cultural, por lo que la cultura tiene un papel importante en la motivación o desmotivación para que los individuos consuman alcohol, en especial los hombres, quienes hacen uso de esta sustancia como método de afrontamiento y es más aceptado socialmente (Palacios, 2012; Mphele, Gralewski \& Balogun, 2013). Por otro lado, el alcohol es metabolizado de manera diferente por hombres y mujeres, lo que le permite a los hombres consumir mayores cantidades en comparación con las mujeres debido a factores como la cantidad de agua en el cuerpo y la distribución de grasas por lo que el límite sería de máximo tres a cuatro unidades de alcohol al día para los hombres y de dos a tres para las mujeres; estos hace que la tolerancia al alcohol sea menor en las mujeres (Gómez, Arnal, Martínez \& Muñoz, 2011; González \& Santolaria, 2011).

Estos resultados permiten comprender mejor la magnitud del problema los datos obtenidos en este estudio nos indican que el estrés es uno de los factores de riesgo más comunes entre la población estudiantil el cual está altamente asociado con el consumo de alcohol como medio de afrontamiento y con la depresión como respuesta a niveles elevados y constantes de estrés negativo, no sólo en universitarios sino también en el nivel medio superior, ya que está es una población vulnerable a estos factores de las cuales se carece de información.

Por lo cual es importante considerar este análisis para posibles intervenciones preventivas que busquen la modificación de las conductas de afrontamiento en los estudiantes y reducir los niveles de depresión y de consumo de alcohol o de estudios explicativos que permitan saber cómo es que se comportan estos factores de acuerdo a las características sociodemográficas de la población, la influencia de la comunidad y el ambiente en el nivel de depresión de los estudiantes.

\section{References:}

1. Aguillón, E. (2014). Alcoholismo como factor de riesgo para el desarrollo de conducta alimentaria de riesgo (Tesis de Licenciatura) Universidad Nacional Autónoma de México. Recuperado de TESIUNAM. 
2. Alcalá, B. (2014). Depresión Autoestima y Género en mujeres adultas: Propuesta de intervención (Tesina de Licenciatura) Universidad Nacional Autónoma de México. Recuperado de TESIUNAM.

3. Alawad, A. \& Slamah, A. (2014). The prevalence of stress among interior design and furniture students. European Scientific Journal, 10(23), 60-72.

4. American Psychiatric Association. (2014). Diagnostic and statistical manual of mental disorders (5a. ed.).Washington, DC: Autor.

5. American Psychiatric Association. (2014). Encuesta de la Asociación Americana de Psicología demuestra que el estrés en los adolescentes es similar al de los adultos. Recuperado de http://www.apa.org/news/press/releases/2014/02/estresadolescentes.aspx

6. Armendáriz, N., Villar, M., Alonso, M., Alonso, B. \& Oliva, N. (2012). Eventos estresantes y su relación con el consumo de alcohol en estudiantes universitarios. Investigación en Enfermería: Imagen y Desarrollo, 14(2), 97-112.

7. Barnett, B. (2013). ¿¡Salud!? ¿Realmente el consumo de alcohol ayuda a aliviar el estrés? El uso del alcohol para relajarse solo funciona exitosamente para ciertas personas en cantidades y circunstancias específicas. Recuperado el 24 de julio de 2016, de de http://expansion.mx/salud/2013/09/26/salud-realmente-el-consumode-alcohol-ayuda-a-aliviar-el-estres

8. Berrío, N. \& Mazo, R. (2011). Estrés académico. Revista de psicología Universidad de Antioquia, 3(2), 66-82.

9. Centro de Estudios para el Adelanto de las Mujeres y la Equidad de Género (2013). Diagnóstico estadístico sobre la depresión y el estrés en las mujeres mexicanas. Recuperado el 7 de agosto de 2016, de http://archivos.diputados.gob.mx/Centros_Estudio/ceameg/ET_2013/ 04_SMDES.pdf

10. Chávez, M., Contreras, O. \& Velázquez, M. (2014). Afrontamiento y depresión: su relación con pensamiento constructivo y no constructivo en universitarios. Revista Intercontinental de Psicología y Educación, 16(2), 31-49.

11. Cubillas, M., Román, R., Valdez, E. \& Galaviz, A. (2012). Depresión y comportamiento suicida en estudiantes de educación media superior en Sonora. Salud mental, 35, 45-50.

12. Flórez, I. \& Trujillo, A. (2013). ¿Tomar no tomar?: análisis de los motivos de consumo y no consumo de alcohol en jóvenes. Revista diversitas-perspectivas en psicología, 9(1), 199-215. 
13. García, A. \& Escalera, M. (2011). El estrés académico ¿una consecuencia de las reformas educativas del nivel medio superior?. Revista electrónica de Psicologíaa Iztacala, 14(3), 156-175.

14. Gómez, J., Arnal, A., Martínez, A. \& Muñoz, D. (2011). Mujeres y uso del alcohol en las sociedades contemporáneas. Revista Española de Drogodependencias, 35(3), 273-284.

15. González- González, A., Juárez, F., Solís, C., González-Forteza, C., Jiménez, A., Medina-Mora, M. \& Fernández-Varela, H. (2012). Depresión y consumo de alcohol y tabaco en estudiantes de bachillerato y licenciatura. Salud mental, 35, 51-55.

16. González, E. \& Santolaria, F. (2011). Repercusiones orgánicas del consumo de alcohol: diferencias entre hombres y mujeres. Medicina Clínica, 137(2), 66-67.

17. González-Olaya, H., Delgado-Rico, H., Escobar-Sánchez, M. \& Cárdenas-Angelone, M. (2014). Asociación entre el estrés, el riesgo de depresión y el rendimiento académico en estudiantes de los primeros semestres de un programa colombiano de medicina. Fundación Educación Médica, 17(1), 47-54.

18. Instituto Nacional de Salud Pública. (2012). Consumo de alcohol en México, 2000-2012: estrategias mundiales para reducir su uso nocivo. Encuesta Nacional de Salud y Nutrición. Recuperado de http://ensanut.insp.mx/doctos/analiticos/ConsumoAlcohol.pdf

19. Instituto Nacional de Psiquiatría Ramón de la Fuente Muñíz INPRFM (2012). Encuesta Nacional de Adicciones 2011: Reporte de Alcohol. México: Instituto Nacional de Psiquiatría Ramón de la Fuente Muñíz/ secretaria de Salud.

20. Iqbal, S., Gupta, S. \& Venkatarao, E. (2015). Stress, anxiety \& depression among medical undergraduate students \& their sociodemographic correlates. Indian J Med Res, 141, 354-357.

21. Jiménez, A., Wagner, F., Rivera, M. \& González-Forteza, C. (2015). Estudio de la depresión en estudiantes de la Ciudad de México y del Estado de Michoacán por medio de la versión revisada de la CES-D. Salud Mental, 38(2), 103-107.

22. Khurshid, S., Parveen, Q., Yousuf, M. \& Ghafoor, A. (2015). Effects of depression on students' academic performance. Science International, 27(2), 1619-1624.

23. Lazarus, R. \& Folkman, S. (1986). Estrés y procesos cognitivos. España: Ediciones Martínez Roca.

24. Matud, M., Correa, M., Bethencourt, J. \& Del Pino, M. (2014). Relevancia del estatus menopáusico en las diferencias en salud mental entre mujeres y hombres. Anales de Psicología, 30(3), 927936. 
25. Maturana, H. \& Vargas, S. (2015). Estrés escolar. Revista Médica Clínica Condes, 26(1), 34-41.

26. Mphele, S., Gralewski, C. \& Balogun, S. (2013). Stress and Alcohol Use Among College Students: A Case of Molepolole College. Students Journal Of Humanities And Social Science, 8(3), 1-6.

27. Organización Mundial de la Salud (2012). La Depresión. Recuperado el 7 de julio de 2016, de http://www.who.int/mediacentre/factsheets/fs369/es/

28. Organización Mundial de la Salud, (2015). Alcohol. Recuperado el 7 de julio de 2016, de http://www.who.int/mediacentre/factsheets/fs349/es/

29. Palacios, J. (2012). Exploración de los motivos para consumir alcohol en adolescentes. Psicología Iberoamericana, 20(1), 29-39.

30. Tovolacci, M., Ladner, J., Grigioni, S., Richard, L., Villet, H. \& Dechelotte, P. (2013). Prevalence and association of perceived stress, substance use and behavioral addictions: a cross-sectional study among university students in France, 2009-201. Bio Med Central Public Health, 13(724), 1-8.

31. Pozos-Radillo, B., Preciado-Serrano, M., Plascencia, A., AcostaFernández, M. \& Aguilera, M. (2015). Estrés académico y síntomas físicos, psicológicos y comportamentales en estudiantes mexicanos de una universidad pública. Estrés y Ansiedad, 21(1), 35-42.

32. Reyes, L., Ibarra, D., Torres, M. \& Razo, M. (2012). El estrés como un factor de riesgo en la salud: análisis diferencial entre docentes de universidades públicas y privadas. Revista Digital Universitaria, 13(7), 3-14.

33. Sandoval, U., Torres, R., \& Martínez, J. (2015). Consumo Riesgoso de Alcohol en Estudiantes de la Universidad La Salle Ciudad de México. Memorias del XVII concurso lasallista de investigación, desarrollo e innovación, 2, 53-57.

34. Soria, R., Ávila, E. \& Morales, A. (2014). Depresión y problemas de salud en estudiantes universitarios de la carrera de Medicina. Diferencias de género. Alternativas en Psicología, 31, 45-59.

35. Telumbre-Terrero, J., Esparza-Almanza, S., Alonso-Castillo, B. \& Alonso-Castillo, M. (2016). Consumo de alcohol y tabaco en estudiantes de enfermería. Enfermería Actual en Costa Rica, 30, 116.

36. The American Institute of Stress (2012). Stress is Kiling You. Recuperado el 2 de julio de 2016, de http://www.stress.org/stress-iskilling-you/ 
37. Uribe, J., Verdugo, J. \& Zacarías, X. (2011). Relación entre percepción de riesgo y consumo de drogas en estudiantes de bachillerato. Psicología y Salud, 21(1), 47-55.

38. Veytia, M., González, N. \& Andrade, P. (2012). Depresión en adolescentes: El papel de los sucesos vitales estresantes. Salud mental, 35(1), 37-43.

39. Vega, C., Villagrán, M., Nava, C. \& Soria, R. (2013). Estrategias de afrontamiento, estrés y alteración psicológica en niños. Alternativas en Psicología, 28, 92-105. 